\title{
Métodos de cuantificación de la carga de entrenamiento en deportes de resistencia cíclica
}

\author{
Methods of quantification of the training load in cyclical endurance sports
}

Muñoz Pérez Iker, Ph.D.

Corporación Universitaria del Caribe (CECAR). Ciencias de la Actividad Física y del Deporte. Sincelejo, Colombia. Correspondencia: iker.munoz@cecar.edu.co

\section{Resumen.}

El presente trabajo tiene como objetivo el realizar un estado del arte con respecto a los principales sistemas y propuestas de cuantificación de cargas de entrenamiento $(\mathrm{TL})$ en deportes de resistencia cíclica. En primer lugar, se expusieron los métodos propuestos para la cuantificación de la carga en función de la percepción subjetiva del esfuerzo. En segundo lugar, se presentaron los diferentes métodos encontrados en la bibliografía científica basados en variables fisiológicas. Finalmente, se presentó futuras aplicaciones prácticas sobre las posibilidades que ofrecen las nuevas tecnologías ofertadas por el mercado, en lo que a la elaboración de nuevos sistemas de cuantificación de la TL se refiere.

Palabras clave: Cuantificación de cargas de entrenamiento, frecuencia cardíaca, intensidad de ejercicio.

\begin{abstract}
.
The aim of this study was to make a state of the art regarding the main methods and purposes of training load (TL) quantification in endurance cyclic sports. Firstly, it was presented the methods for quantifying the TL based on the perceived exertion. Secondly, it was shown the different methods found in the scientific references which were based on physiological variables. Finally, it was presented future practical applications regarding the possibilities which new technologies presently on the market offer, in the development of new systems of TL quantification.
\end{abstract}

Key words: Training load quantification, heart rate, exercise intensity. 


\section{Introducción}

El objetivo final del entrenamiento deportivo: promover un mayor rendimiento, es un proceso adaptativo que se da durante un tiempo determinado. Para conseguir este objetivo es necesario una correcta progresión, distribución y optimización en las cargas de entrenamiento (TL) (Mujika, 1998, Mujika \& Padilla, 2003). La medición de la carga de entrenamiento (relativa o absoluta) es un elemento clave a la hora de poder valorar y comparar diferentes sistemas de entrenamiento y por consiguiente, medir las adaptaciones que provocan estos.

La cuantificación del entrenamiento ha sido objeto de estudio de numerosas investigaciones (Banister \& Calvert, 1980; Morton, Fitz-Clarke \& Banister, 1990; Mujika et al. 1996; Lucia, Hoyos, Carvajal y Chicharro, 1999; Borresen \& Lambert, 2007; Hayes \& Quinn, 2009; Manzi, Iellamo, Impellizzeri, D'Ottavio, \& Castagna, 2009; Stagno, Thatcher, \& van Someren, 2007; Wood, Hayter, Rowbottom \& Stewart 2005; Sylta, Tønnessen, \& Seiler, 2014; Borresen, \& Lambert, 2009.) las cuales han propuesto diferentes métodos $y$ variables de estudio para arrojar luz sobre el proceso de cuantificación. Sin embargo, la mayoría de los entrenadores deportivos, sigue utilizando para cuantificar el entrenamiento métodos subjetivos, lo cual conlleva un riesgo para la salud del deportista al no ser monitoreado de manera alguna su estado fisiológico (Cejuela \& EsteveLanao, 2011).

La presente revisión tuvo por objetivo exponer los principales sistemas y propuestas a la hora de cuantificar la TL en deportes de resistencia cíclica.

Cuantificación en función de la fatiga percibida. La utilización de la percepción del esfuerzo (RPE en inglés Perceived Exertion) fue propuesta en primera instancia por el psicólogo sueco Gunnar Borg y Dahlstrom (1964a; 1964b; Borg, 1975). Este investigador, propuso una escala de percepción en la cual el deportista determina la intensidad del esfuerzo (escala de 6 a 20 puntos o de 0 a 10). La percepción de la fatiga en un momento determinado ha sido correlacionada significativamente con la intensidad del esfuerzo (Singh, Foster, \& McGuigan, 2007).

La utilidad de esta cuantificación subjetiva, fue validada en varios estudios que relacionaron este método (subjetivo) con variables fisiológicas objetivas (Seiler \& Kjerland. 2006; Foster et al. 2001; DellaValle \& Haas, 2013; Borresen, \& Lambert, 2008).

Una de las primeras propuestas de cuantificación en función de criterios subjetivos fue la de Foster et al. (2001). En este trabajo, se sugirió la asignación de una puntuación de la intensidad a la sesión en función de la percepción de atleta (Tabla 1.), posteriormente se deberá multiplicar esta intensidad por el tiempo de duración de la sesión.

Un ejemplo de la cuantificación del entrenamiento sería el siguiente: una percepción de la fatiga de 7 por 20 minutos de duración, esto representaría

Tabla 1. Escala de percepción del esfuerzo

\begin{tabular}{cc}
\hline Puntuación & Descripción \\
\hline 0 & Descanso \\
1 & Muy, muy fácil \\
2 & Fácil \\
3 & Moderado \\
4 & Algo duro \\
5 & Duro \\
6 & - \\
7 & Muy duro \\
8 & - \\
9 & Máximo \\
10 & \\
\hline
\end{tabular}

Fuente: Modificada de Foster et al., 2001 
un índice de TL total de 140. Uno de los principales logros de este método de cuantificación es la capacidad de calcular tanto el entrenamiento de resistencia como el trabajo de fuerza. Posibilitando de esta manera una cuantificación de todos los factores que forman parte de la preparación de un atleta.

Por otra parte, una de las principales desventajas de este método es la necesidad de un entrenamiento - familiarización previa con esta escala para obtener unas mediciones fidedignas. Otra desventaja del método propuesto por Foster et al. (2001) es la consideración del tiempo total de duración de la sesión, incluyendo pausas, omitiendo el cálculo de la densidad, y englobando la cuantificación del entrenamiento de fuerza a través de la variable "tiempo", lo cual no tiene mucho sentido para cuantificar la fuerza (Cejuela \& Esteve-Lanao, 2011).

Uno de las últimas investigaciones publicadas respecto a la cuantificación subjetiva de la TL (Cejuela \& EsteveLanao, 2011) presenta una escala de puntuación con valores de 0 a 5 (incluyendo incrementos de 0,5).

Los autores de este método (ECS: Equivalente de Carga Subjetiva), alegan la imposibilidad de controlar todos los factores del entrenamiento (entrenamiento, fatiga acumulada, estado nutricional) y las diferentes formas de cuantificar las distintas cualidades físicas (Cejuela \& EsteveLanao, 2011). El método ECS propone la medición de valores diarios y acumulados, a comparar con la evolución de la carga objetiva, debiendo ser esta gradualmente creciente, mientras que la subjetiva debería permanecer más estable 0 decreciente. La principal limitación de este método es que está todavía por validar la medición de la carga subjetiva (ECS).

\section{Impulso de entrenamiento \\ (TRIMP). El modelo denominado}

Impulso de Entrenamiento (TRIMP del inglés Training Impulse), cuantifica el estímulo de entrenamiento como una composición de carga externa y carga interna, multiplicando la TL (volumen) por la intensidad del entrenamiento (Taha \& Scott, 2003).

Bannister (1980) propuso por primera vezeste método, basado en el incremento de la FC gradualmente ponderada. En la figura 1 se muestra el cálculo del sistema propuesto por Bannister en el cual la duración (en min.) se multiplica por un factor de intensidad diferente para hombre o mujer.

Figura 1. Sistema original TRIMP

TRIMP $=$ duración del entrenamiento $(\mathrm{min}) \times$ (factor $\mathrm{A}$ $x \Delta \mathrm{FC} \times \exp ($ factor $\mathrm{B} \times \Delta \mathrm{FC}$ )

Ratio $\Delta F C=$ (FC media durante el ejercicio - FC media durante la recuperación)/(FCmáx. durante el ejercicio

- FC media durante la recuperación)

Factor $\mathrm{A}=0,86$ y Factor $\mathrm{B}=1,67$ para mujeres

Factor $A=0,64$ y Factor $B=1,92$ para hombres

Fuente: Bannister, 1980

Basándose en el sistema TRIMP (Bannister, 1980), una mujer que haya entrenado $20 \mathrm{~min}$ con una Frecuencia Cardíaca máxima (FC máx.) de 165 pulsaciones, una FC media de 160 pulsaciones y una FC media durante la recuperación de 120 pulsaciones obtendrá una TL de 65,8 TRIMPs.

Las limitaciones para este modelo se observan en una imposibilidad de cuantificar las intensidades por encima de la FC máx. Ias propias de la utilización de la FC (Achten \& Jeukendrup, 2003) y la falta de medición de las pausas.

Morton et al. (1990) sugirieron una modificación de la propuesta de Bannister, con el objetivo de poder cuantificar de una mejor manera las intensidades altas (Figura 2.). 
Figura 2. Modificación de sistema TRIMP

TRIMP = duración del entrenamiento $(\mathrm{min})$ $\Delta \mathrm{FC} \cdot 2,718 \exp ($ factor $\mathrm{B} \cdot \Delta \mathrm{FC}$ )

Fuente: Morton et al., 1990

Otra de las propuestas que se ha realizado, en los últimos años, basándose en el estudio de Bannister (1980) ha sido la del sistema TRIMPi (estímulo de entrenamiento individualizado en castellano). Manzi et al. (2009), con esta modificación, quisieron evitar la desproporcionada importancia que se da a los entrenamientos de larga duración y poca intensidad comparado con los entrenamientos más intensos pero de duración corta (Manzi et al. 2009). Para ello este grupo propone multiplicar la $\triangle F C$ por un factor (y) para reflejar la intensidad del esfuerzo. Este factor (y) se basa en función del incremento exponencial de los niveles de concentración de lactato sanguíneo (bLA) y la elevación de la FC desde el reposo (Figura 3.).

Figura 3. Modificación de sistema TRIMP

TRIMP $=$ tiempo $(\min ) \cdot \Delta \mathrm{FC} \cdot \mathrm{y}$
$\Delta \mathrm{FC}=(\mathrm{FC}$ durante el ejercicio $-\mathrm{FC}$ en reposo/ $\mathrm{FCmáx}-\mathrm{FC}$ en reposo $)$
$y=0,64^{\mathrm{eb}(\mathrm{c}) \mathrm{x}}$
$\mathrm{e}=$ basado en el logaritmo neperiano, $\mathrm{x}=\Delta \mathrm{FC}$,
$\mathrm{b}=0,64$ para hombres y $\mathrm{c}=1,92$ para mujeres

Fuente: Manzi et al., 2009

Uno de los modelos matemáticos más novedosos a la hora de cuantificar el entrenamiento fue el presentado por Hayes y Quinn (2009). Este modelo permite realizar comparaciones tanto para entrenamientos continuos como interválicos y toma en consideración las pausas y el tipo de recuperación de estas (figura 4.). El principal problema de este sistema es su complejidad a la hora de cuantificar las cargas, además de no haber sido testado todavía con pruebas de campo (Cejuela \& EsteveLanao, 2011).
Figura 4. Sistema de puntuación (W) TRIMPS

$$
\begin{aligned}
& \begin{array}{l}
W=I\left(D_{\text {act }}\right) \times C\left(D_{\text {act }}\right) \times D \\
=\left(\frac{v_{\text {act }}}{v_{\text {opt }}}+\frac{v_{\text {act }}-v_{\text {crit }}}{v_{\text {max }}-v_{\text {crit }}}\right) \times\left(1+\frac{I\left(n D_{\text {act }}\right)-I\left(D_{\text {act }}\right)}{I\left(D_{\text {act }}\right)} \mathrm{e}^{-\sigma_{1} \frac{\tau_{\text {rec }}}{\text { effort }}}\right) \\
\quad \times n D_{\text {act }}\left(q_{D}+\left(q_{n D}-q_{D}\right) \mathrm{e}^{-\frac{\tau_{\text {rec }}}{\tau_{\text {effort }}}}\right)
\end{array} \\
& \text { I = intensidad de la sesión, } \mathrm{C}=\text { densidad de la sesión, D = volumen de } \\
& \text { la sesión. } \\
& \text { Fuente: Hayes \& Quinn, 2009. }
\end{aligned}
$$

Propuestas como la desarrollada por Lucía et al. (1999), la cual trata de simplificar el modelo original de Bannister, han mostrado su utilidad. Basándose en el modelo trifásico de Skinner y y McLellan (1980), se redujeron las zonas de entrenamiento a 3 zonas. La primera zona se situó por debajo del primer umbral ventilatorio (VT1), la segunda zona se da entre umbrales, y la tercera zona se desarrolla a intensidades mayores del segundo umbral ventilatorio (VT2). A cada minuto en una de ellas se le asignará una puntuación, siendo 1 punto por minuto en zona 1, 2 por minuto en zona 2 y 3 por minuto en zona 3 . Para obtener la carga total de la sesión solamente se tendrá que sumar la puntuación obtenida en cada zona.

La gran simplicidad del método aporta una mayor rapidez a la hora de cálculos matemáticos y en la recogida de los datos, varios estudios científicos usaron esta modificación del sistema TRIMP propuesta por Lucía et al. (1999) para cuantificar la TL en corredores de resistencia (Muñoz, Seiler, Alcocer, Carr, \& Esteve-Lanao, 2015; Muñoz, Seiler, Bautista, España, Larumbe, \& Esteve-Lanao, 2014) y en triatletas participantes en un $1 / 2$ Ironman (Muñoz, Cejuela, Seiler, Larumbe, \& EsteveLanao, 2014). No obstante, es necesario destacar que este sistema está sujeto a la variación de la FC con las limitaciones que esto puede conllevar a la hora de medir el estrés psíquico y fisiológico del organismo (Achten y Jeukendrup, 2003). Otro factor a tener en cuenta, es la igualdad en las puntuaciones a 
Muñoz. Métodos de cuantificación en deportes de resistencia cíclica

intensidades fisiológicas diferenciadas. Un ejemplo de ello podría ser la asignación, mediante este método, de la misma puntuación a un minuto desarrollando una intensidad igual o ligeramente superior al VT2 y un minuto a intensidades próximas al $\mathrm{VO}_{2}$ máx.

Recientemente se ha publicado una nueva propuesta para la cuantificación de la $T L$ en nadadores de élite internacionales (García-Ramos et al. 2015) basándose en el sistema TRIMP propuesto por Bannister (1980). Este método denominado Impulsos de Entrenamiento Acumulado (TRIMPc) presenta una modificación importante ya que permite tener en cuenta el tiempo de ejercicio y el de pausa o recuperación. De esta manera, se incrementa la puntuación obtenida en función del ratio actividad-recuperación (comparando la misma actividad con el sistema de Bannister), permitiendo de esta manera corregir la tendencia del sistema de Bannister a subestimar la TL debido a que no discrimina entre el ejercicio y la pausa durante un entrenamiento interválico (GarcíaRamos et al. 2015).

Exceso de Consumo de Oxígeno Post-Ejercicio (EPOC). La medición del EPOC ha sido sugerida como reflejo de la respuesta del organismo a una sesión de entrenamiento (Jobson, Passfield, Atkinson, Barton, \& Scarf, 2009). Como con las mediciones del $\mathrm{VO}_{2}$ y del bLA, el EPOC necesita ser medido en el laboratorio, lo que conlleva un gasto importante en equipamiento adecuado, un coste de tiempo considerable y una duración de los resultados variable.

Rusko et al. (2003) (citado en CejuelaAnta \& Esteve-Lanao, 2011) propusieron un modelo matemático capaz de estimar el EPOC en función de la FC (Figura 5.). El cálculo de este modelo es relativamente complejo y requiere de un software y hardware específico (Suunto $^{\mathrm{TM}}$ t6 HeartRate monitor).
Figura 5. Modelo matemático para cuantificar el EPOC basado en FC

$\operatorname{EPOC}(t)=f\left(\operatorname{EPOC}_{(\mathrm{t}-1)}, \% \mathrm{VO}_{2}\right.$ máx, $\left.\Delta t\right)$

$\operatorname{EPOC}(\mathrm{t})=$ es calculado usando la intensidad de ese momento ( $\% \mathrm{VO}_{2} \mathrm{max}$ ), duración del ejercicio (tiempo entre dos puntos $(\Delta t)$ y el EPOC en la muestra anterior $\left(\mathrm{EPOC}_{(\mathrm{t}-1)}\right)$

Fuente: Rusko et al., 2003

Variabilidad de la Frecuencia Cardíaca. El uso de la FC como herramienta útil para medir la intensidad de entrenamiento y en especial, la variabilidad de esta para estimar la regulación cardíaca del sistema nervioso autónomo (SNA) y su perturbación ante el estrés fisiológico y psíquico ha sido ampliamente estudiado (Aubert, Seps, \& Beckers, 2003; Chandola, Heraclides, \& Kumari, 2010).

El estímulo que provoca una sesión de entrenamiento influirá directamente sobre el SNA, el cual jugará un papel clave en las respuestas individuales al entrenamiento de cada sujeto ante la magnitud de dicho estímulo (Chalencon et al., 2012; Hautala, Kiviniemi, \& Tulppo, 2009).

El uso de la variabilidad de la FC (VFC) post-ejercicio ha tomado mayor protagonismo en los últimos años ante la comunidad científica como un método válido para cuantificar la TL (Kaikkonen, Hynynen, Mann, Rusko, \& Nummela, 2010; 2012; Kaikkonen, Nummela, \& Rusko, 2007; Martinmäki \& Rusko, 2008).

Respecto al uso de la VFC para la cuantificación del entrenamiento, un estudio realizado por Saboul, Balducci, Millet, Pialoux y Hautier (2015) presentó un sistema basado en la medición de esta variable (Figura 6.). Estos autores propusieron una nueva forma de cuantificar el rendimiento llamada Índice de Variabilidad de la Frecuencia Cardíaca ( $\mathrm{TL}_{\text {HRV }}$ en inglés Training Load Index of Heart Rate Variability). Mediante el 
cálculo de este índice (observar Figura 6. para la determinación de este índice) la TL podrá cuantificarse teniendo en cuenta tanto los entrenamientos continuos como interválicos. Una de las principales novedades que ofrece este trabajo es que se tuvo en cuenta el intervalo de tiempo de la onda R-R (siendo uno de los parámetros más representativos para medir el ajuste en la actividad vagal) (Buchheit, Laursen, \& Ahmaidi 2007; Buchheit et al., 2010), por lo que permite equiparar y cuantificar entrenamientos continuos con interválicos.

Figura 6. Modelo matemático para la cuantificación del Índice de Variabilidad de la Frecuencia Cardíaca.

\section{$\mathrm{TL}_{\text {HRV }}=\mathrm{T} \mathrm{x}$ Pre5 - Post 5 Post30 - Post5}

$\mathrm{TL}_{\mathrm{HRV}}=$ índice de carga de entrenamiento; $\mathrm{T}=$ duración (en minutos) del entrenamiento; Pre5 = valor de medición de la HRV 5 minutos antes del calentamiento de la sesión (mseg); Post5 = valor de la medición de la HRV 5 minutos después de la sesión (mseg); Post30 = valor de la medición de la HRV 30 minutos después de la sesión (mseg)

Fuente: Saboul et al., 2015

Mediante el uso de la VFC puede medirse y cuantificarse de manera más precisa la incidencia de un entrenamiento interválico de alta intensidad y corta duración, haciendo una estimación de la TL más próxima a la realidad que con los sistemas anteriormente expuestos (Bannister,1980; Foster et al. 2001). No obstante, la necesidad de realizar las mediciones después del ejercicio, en un determinado entorno, bajo unas condiciones específicas, con una hidratación determinada, sin producirse una ingesta de comida y la necesidad de un sistema de monitorización que permita medir la VFC, en ocasiones, podrá resultar un tanto dificultoso.

Producción de potencia (W). La irrupción en el mercado de sistemas que permiten una medición continua de la potencia generada $\left(\mathrm{SRM}^{\mathrm{TM}}\right.$ y Power $\operatorname{Tap}^{\mathrm{TM}}$ ) y la utilización por parte de ciclistas de élite durante el entrenamiento y competición de estos sistemas, ha incrementado el interés de investigadores por este tipo de mediciones (Elbert et al. 2005; Elbert, Martin, Stephens \& Withers, 2006).

La asociación de una determinada intensidad (W) a unas respuestas fisiológicas, permiten el análisis tanto del entrenamiento como de la competición (Jobson et al. 2009; Gardner, Stephens, Martin, Lawton, Lee, \& Jenkins, 2004) sin depender de la variabilidad de la FC. Una de las aproximaciones para salvar la dificultad de interpretar los datos obtenidos por estos sistemas, ha sido la propuesta por Elbert et al. (2005), que aboga por evaluar el tiempo total pasado por el atleta en una zona determinada (zonas de intensidades distintas, medidas en W).

Zonas de entrenamiento. La utilización de escalas de entrenamiento en función de la FC, velocidad o bLA ha sido ampliamente propuesta por diversos autores. La problemática para estas escalas se presenta en la medición de intensidades superiores al $\mathrm{VO}_{2}$ máx. Autores como, Edwards (1993) han realizado otro tipo de aportaciones basándose en la distribución de diferentes zonas de entrenamiento ( 5 zonas en concreto) en función de la FC (zona 1: 50-60\% FC máx.; zona 2: 6070\% FC máx.; zona 3: 70-80\% FC máx, zona 4: 80-90\% FC máx. y zona 5: 90$100 \%$ FC máx.). A cada zona le asigna una puntuación de 1 a 5, obteniendo la Zona 1 un punto y la quinta zona 5 .

Para obtener la cuantificación de la carga, Edwards propone la multiplicación del tiempo (min.) pasado en cada zona por la puntuación de esta misma. Un ejemplo de este sistema sería: $20 \mathrm{~min}$ en zona $2+10$ min en Zona $3+1$ min en zona 5 , lo cual haría una puntuación total de 75 .

Las limitaciones a este modelo son las propias de la utilización de la FC (Achten \& Jeukendrup, 2003), no mide la densidad, solo sirve para entrenamientos continuos (sin pausas), 
Muñoz. Métodos de cuantificación en deportes de resistencia cíclica

y no permite la cuantificación de las intensidades por encima de la FC máx. Unos de los métodos propuestos en la literatura científica, es el desarrollado por Mujika et al. (1996). La introducción del concepto de unidades de entrenamiento basadas en la bLA, evita la dependencia de la FC y las limitaciones que esto conlleva (Tabla 2.).

Tabla 2. Unidades de entrenamiento

\begin{tabular}{cc}
\hline Nivel de intensidad & Coeficiente de multiplicación \\
\hline I & 1 \\
II & 2 \\
III & 3 \\
IV & 5 \\
V & 8 \\
\hline
\end{tabular}

Las zonas de intensidad I, II, III representan velocidades de nado inferiores $(\sim 2 \mathrm{mMol} / \mathrm{l})$, igual $\left(\sim 4 \mathrm{mMol} \cdot \mathrm{L}^{-1}\right)$ y ligeramente superior $(\sim 6$ $\left.\mathrm{mMol} \cdot \mathrm{L}^{-1}\right)$. La zona IV fue definida como de alta intensidad $\left(\sim 10 \mathrm{mMol} \cdot \mathrm{L}^{-1}\right)$ y la zona $V$ como de intensidad máxima.

Fuente: Mujika et al., 1996

Para obtener el cálculo de la $T L$, es necesario multiplicar el volumen $(\mathrm{km})$ en cada zona de intensidad por su coeficiente, sumando todas las puntuaciones para conseguir la carga total.

Basándose en una escala que represente más fielmente las diferentes zonas e intensidades de entrenamiento, Cejuela y Esteve-Lanao (2011) proponen el modelo ECOs (Equivalentes de la Carga Objetivo). Este modelo permite la cuantificación en un deporte complejo como es el triatlón.

Este sistema propone la asignación de unos valores en función de la zona de entrenamiento. Esta zona variará su puntuación dependiendo de la intensidad desarrollada en ella (menos intensidad menor puntuación). Los autores de este modelo abogan por una cuantificación individualizada para establecer el tiempo límite en cada intensidad basándose en la eficiencia, velocidad crítica o el IR, estableciendo de esta manera los valores de las zonas (Tabla 3.).
Tabla 3. Zonas y puntuación de las zonas del modelo ECOs

\begin{tabular}{ccc}
\hline Zona & Carrera & Valor \\
\hline 1 & <UAE & 1 \\
2 & UAE & 2 \\
3 & UAE-UAN & 3 \\
4 & UAN & 4 \\
5 & >UAN & 6 \\
6 & VAM & 9 \\
7 & Cap LAC & 15 \\
8 & Pot LAC & 50 \\
\hline
\end{tabular}

$<U A E$ : Umbral Aeróbico (primer umbral fisiológico); UAE: Umbral Aeróbico; UAE-UAN: entre umbrales; UAN: Umbral Anaeróbico (segundo umbral fisiológico): >UAN: intensidad entre UAN Y PAM. PAM: Potencia Acŕbica Máxima; Láctica.Fuente: Cejuela \& Esteve-Lanao, 2011

La ponderación de la carga es obtenida tras multiplicar el tiempo (min) por el valor de la zona donde ha entrenado. A su vez, este resultado ha de multiplicarse por 0,75 en la natación y por 0,5 en ciclismo (la carrera se tomará como referencia asignándole el valor de 1 ).

La cuantificación de la densidad del entrenamiento, en especial si se trata de entrenamientos continuos, surge como limitante de este método. En la actualidad este sistema ha sido utilizado en varios estudios científicos por lo que permite cuantificar de manera óptima la TL (Debevec et al. 2015; Medina et al. 2015; Villaño et al. 2015).

Futuras aplicaciones prácticas. Es deseable que la cuantificación del entrenamiento no tome variables únicas y estrictamente objetivas, aunque hasta la actualidad pocos han sido los estudios publicados que incluyan para la cuantificación de la TL las variables subjetivas y objetivas (Foster et al. 2001; Cejuela \& Esteve-Lanao, 2011). Es obvio, que las variables que determinan una sesión de entrenamiento (volumen, intensidad y densidad) han de ser medidas y cuantificadas para su manipulación. No obstante, la capacidad de percepción del sujeto aporta una información valiosa y efectiva tanto para determinar la intensidad del ejercicio, como para conocer la percepción de fatiga acumulada ello posibilita un ajuste casi inmediato de la TL. El poder dar un feedback inmediato al entrenador sobre 
la intensidad de entrenamiento que él ha prescrito al atleta (carga objetiva), y la percepción del esfuerzo por parte del atleta (carga subjetiva) posibilitará un ajuste óptimo de la TL.

La disponibilidad de mecanismos de monitorización y medición de las variables fisiológicas asociadas a la intensidad del entrenamiento jugarán un papel clave en la elección de un método de cuantificación u otro. En la actualidad, es posible encontrar en el mercado productos accesibles para la medición continua de la VFC y la potencia o velocidad generada. Esta irrupción de productos tecnológicos posibilita la medición de variables fisiológicas (VFC y potencia o velocidad generada) en situaciones de entrenamiento $y$ competición reales, las cuales hasta hace unos años solo eran susceptibles de ser medidas en un laboratorio. Por ello, el uso de estas nuevas tecnologías posibilitará nuevas propuestas para cuantificación de la TL como la realizada por Saboul et al. (2015).

Pese a no haber un modelo perfecto de cuantificación de la TL en deportes de resistencia cíclicos, sí es de vital importancia el uso de alguno de ellos con el fin de aportar un mayor control sobre el proceso de entrenamiento. Siendo parte fundamental para la comparación de cargas de trabajo obtenidas sobre el mismo atleta en sesiones y periodos de preparación distintos, o entre diferentes atletas.

\section{Referencias}

Achten, J., \& Jeukendrup, A. E. (2003). Heart rate monitoring: Applications and limitations. Sports Medicine (Auckland, N.Z.), 33(7), 517-538.

Aubert, A. E., Seps, B., \& Beckers, F. (2003). Heart rate variability in athletes. Sports Medicine, 33, 889-919

Banister, E. W., \& Calvert, T. W. (1980). Planning for future performance: Implications for long term training. Canadian Journal of Applied Sport Sciences.Journal Canadien Des Sciences Appliquées Au Sport, 5(3), 170-176.

Borg, G. \& Dahlstrom, H. (1964a). A case study of perceived exertion during a work test. Acta Societatis Medicorum Upsaliensis, 67, 91-93.

Borg, G. \& Dahlstrom, H. (1964b). A pilot study of perceived exertion and physical working capacity. Acta Societatis Medicorum Upsaliensis, 67, 21-27.
Borg, G. A. (1975). Perceived exertion. Exercise and Sport Sciences Reviews, 2, 131-153.

Borresen, J., \& Lambert, M. I. (2007). Changes in heart rate recovery in response to acute changes in training load. European Journal of Applied Physiology, 101(4), 503-511.

Borresen, J., \& Lambert, M.I. (2008). Quantifying training load: a comparison of subjective and objective methods. International Journal of Sports Physiology and Performance, 3(1):1630.

Borresen, J., \& Lambert, M.I. (2009). The quantification of training load, the training response and the effect on performance. Sports medicine (Auckland, N.Z.), 39(9), 779-795. 
Muñoz. Métodos de cuantificación en deportes de resistencia cíclica

Buchheit, M., Chivot, A., Parouty, J., Mercier, D., Al Haddad, H., Laursen, P. B., \& Ahmaidi, S. (2010). Monitoring endurance running performance using cardiac parasympathetic function. European Journal of Applied Physiology, $108,1153-1167$.

Buchheit, M., Laursen, P. B., \& Ahmaidi, S. (2007). Parasympathetic reactivation after repeated sprint exercise. AJP: Heart and Circulatory Physiology, 293(1), 133-141.

Cejuela Anta, R., \& Esteve-Lanao, J. (2011). Training load quantification in triathlon. Journal of Human Sport \& Exercise, $6(2), 218-232$.

Chalencon, S., Busso, T., Lacour, J.-R., Garet, M., Pichot, V., Connes, P., ... Barthélémy, J. C. (2012). A model for the training effects in swimming demonstrates a strong relationship between parasympathetic activity, performance and index of fatigue. PLoS One, 7(12), e52636.

Chandola, T., Heraclides, A., \& Kumari, M. (2010). Psychophysiological biomarkers of workplace stressors. Neuroscience and Biobehavioral Reviews, 35(1), 5157.

DellaValle, D.M \& Haas, J.A. (2013). Quantification of Training Load and Intensity in Female Collegiate Rowers: Validation of a Daily Assessment Tool. Journal of Strength and Conditioning Research, 27(2),540-548.

Devec, T., Pialoux, V., Saugy, J., Schmitt, L., Cejuela, R., Mury, P., ... Millet, G.P.(2015). Prooxidant/Antioxidant Balance in Hypoxia: A Cross-Over Study on Normobaric vs. Hypobaric "Live High-Train Low". PLoS One, 10(9),e0137957.
Ebert, T. R., Martin, D. T., McDonald, W., Victor, J., Plummer, J., \& Withers, R. T. (2005). Power output during women's world cup road cycle racing. European Journal of Applied Physiology, 95(5-6), 529-536.

Ebert, T. R., Martin, D. T., Stephens, B., \& Withers, R. T. (2006). Power output during a professional men's roadcycling tour. International Journal of Sports Physiology and Performance, 1(4), 324-335.

Edwards, S. (1993). The heart rate monitor book. Sacramento: Fleet Feet Press.

Esteve-Lanao, J., Foster, C., Seiler, S., \& Lucia, A. (2007). Impact of training intensity distribution on performance in endurance athletes. Journal of Strength and Conditioning Research / National Strength \& Conditioning Association, 21(3), 943-949.

Fiskerstrand, A., \& Seiler, K. S. (2004). Training and performance characteristics among norwegian international rowers 1970-2001. Scandinavian Journal of Medicine \& Science in Sports, 14(5), 303-310.

Foster, C., Florhaug, J. A., Franklin, J., Gottschall, L., Hrovatin, L. A., Parker, S., et al. (2001). A new approach to monitoring exercise training. Journal of Strength and Conditioning Research / National Strength \& Conditioning Association, 15(1), 109-115.

Fry, R. W., Morton, A. R., \& Keast, D. (1992). Periodisation of training stress-a review. Canadian Journal of Sport Sciences = Journal Canadien Des Sciences Du Sport, 17(3), 234-240.

García-Ramos, A. et al. (2015). Training load quantification in elite swimmers using a modified version of the training impulse method. European Journal of Sport Science, 15(2), 85-93. 
Gardner, A.S., Stephens, S., Martin,

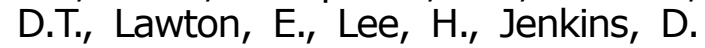
(2004). Accuracy of SRM and power tap power monitoring systems for bicycling. Medicine and Science in Sports and Exercise, 36(7), 1252-1258.

Guellich, A., \& Seiler, S. (2010). Lactate profile changes in relation to training characteristics in junior elite cyclists. International Journal of Sports Physiology and Performance, 5(3), 316-327.

Hautala, A. J., Kiviniemi, A. M., \& Tulppo, M. P. (2009). Individual responses to aerobic exercise: The role of the autonomic nervous system. Neuroscience and Biobehavioral Reviews, 33(2), 107-115

Hayes, P. R., \& Quinn, M. D. (2009). A mathematical model for quantifying training. European Journal of Applied Physiology, 106(6), 839-847.

Jobson, S. A., Passfield, L., Atkinson, G., Barton, G., \& Scarf, P. (2009). The analysis and utilization of cycling training data. Sports Medicine (Auckland, N.Z.), 39(10), 833-844.

Kaikkonen, P., Hynynen, E.,Mann, T., Rusko, H., \& Nummela, A. (2010). Can HRV be used to evaluate training load in constant load exercises? European Journal of Applied Physiology, 108, 435-442.

Kaikkonen, P., Hynynen, E.,Mann, T., Rusko, H., \& Nummela, A. (2012). Heart rate variability is related to training load variables in interval running exercises. European Journal of Applied Physiology, $112,829-838$.

Kaikkonen, P., Nummela, A., \& Rusko, H. (2007). Heart rate variability dynamics during early recovery after different endurance exercises. European Journal of Applied Physiology, 102(1), 79-86.
Luciá, A., Hoyos, J., Carvajal, A., \& Chicharro, J. L. (1999). Heart rate response to professional road cycling: The tour de france. International Journal of Sports Medicine, 20(3), 167-172.

Manzi, V., Iellamo, F., Impellizzeri, F., D'Ottavio, S., \& Castagna, C. (2009). Relation between individualized training impulses and performance in distance runners. Medicine and Science in Sports and Exercise, 41(11), 2090-2096.

Martinmäki, K., \& Rusko, H. (2008). Timefrequency analysis of heart rate variability during immediate recovery from low and high intensity exercise. European Journal of Applied Physiology, 102, 353-360.

Medina, S., Domínguez-Perles, R., CejuelaAnta, R., Villaño, D., Martínez-Sanz, J.M., Gil, P., ... Gil-Izquierdo, A. (2012). Assessment of oxidative stress markers and prostaglandins after chronic training of triathletes. Prostaglandins Other Lipid Media, 99(3-4),79-86.

Morton, R. H., Fitz-Clarke, J., \& Banister, E. W. (1990). Modeling human performance in running. Journal of Applied Physiology (Bethesda, Md.: 1985), 69(3), 1171-1177.

Mujika, I. (1998). The influence of training characteristics and tapering on the adaptation in highly trained individuals: A review. International Journal of Sports Medicine, 19(7), 439-446.

Mujika, I., \& Padilla, S. (2003). Scientific bases for precompetition tapering strategies. Medicine and Science in Sports and Exercise, 35(7), 1182-1187.

Mujika, I., Busso, T., Lacoste, L., Barale, F., Geyssant, A., \& Chatard, J. C. (1996). Modeled responses to training and taper in competitive swimmers. Medicine and Science in Sports and Exercise, 28(2), 251-258. 
Mujika, I., Padilla, S., Pyne, D., \& Busso, T. (2004). Physiological changes associated with the pre-event taper in athletes. Sports Medicine (Auckland, N.Z.), 34(13), 891-927.

Muñoz, I., Cejuela, R., Seiler, S., Larumbe, E., \& Esteve-Lanao, J. (2014). Trainingintensity distribution during an ironman season: relationship with competition performance. International Journal of Sports Physiology and Performance, 9(2),332-339.

Munoz, I., Seiler, S., Alcocer, A., Carr, N., \& Esteve-Lanao, J. (2015). Specific Intensity for Peaking: Is Race Pace the Best Option? Asian journal of sports medicine, 6(3),e24900.

Muñoz, I., Seiler, S., Bautista, J., España, J., Larumbe, E., \& Esteve-Lanao, J. (2014). Does polarized training improve performance in recreational runners? International Journal of Sports Physiology and Performance, 9(2):265272.

Saboul, D., Balducci, P., Millet, G., Pialoux, V., \& Hautier, C. (2015). A pilot study on quantification of training load: The use of HRV intraining practice. European Journal of Sport Science, 16(2),172181.

Seiler, K. S., \& Kjerland, G. Ø. (2006). Quantifying training intensity distribution in elite endurance athletes: Is there evidence for an "optimal" distribution? Scandinavian Journal of Medicine \& Science in Sports, 16(1), 49-56.

Singh, F., Foster, C., Tod, D., \& McGuigan, M. R. (2007). Monitoring different types of resistance training using session rating of perceived exertion. International Journal of Sports Physiology and Performance, 2(1), 34-45.
Skinner, J. S., \& McLellan, T. H. (1980). The transition from aerobic to anaerobic metabolism. Research Quarterly for Exercise and Sport, 51(1), 234-248.

Stagno, K. M., Thatcher, R., \& van Someren, K.,A. (2007). A modified TRIMP to quantify the in-season training load of team sport players. Journal of Sports Sciences, 25(6), 629-634.

Sylta, o., Tønnessen, E., \& Seiler, S. (2014). From heart rate data to training quantification: a comparison of 3 methods of training intensity analysis. International Journal of Sports Physiology and Performance, 9(1):100107.

Taha, T., \& Thomas, S. G. (2003). Systems modelling of the relationship between training and performance. Sports Medicine (Auckland, N.Z.), 33(14), 1061-1073.

Villaño, D., Vilaplana, C., Medina, S., CejuelaAnta, R., Martínez-Sanz, J.M., Gil, P., ... , Gil-Izquierdo, A. (2015). Effect of elite physical exercise by triathletes on seven catabolites of DNA oxidation. Free radical research, 49(8),973-983.

Wood, R. E., Hayter, S., Rowbottom, D., \& Stewart, I. (2005). Applying a mathematical model to training adaptation in a distance runner. European Journal of Applied Physiology, 94(3), 310-316. 